\title{
UNDERSTANDING VS. DESCRIBING OTHERS' MINDS: MENTAL STATE LANGUAGE IN A SMALL-SCALE SOCIETY
}

\author{
ANDREW MARCUS SMITH* ${ }^{* 1}$, ERICA A. CARTMILL ${ }^{1}$, and H. CLARK BARRETT ${ }^{1}$ \\ *Corresponding Author: andrewmsmith@ucla.edu \\ ${ }^{1}$ Department of Anthropology, University of California, Los Angeles, USA
}

Theory of mind, or mentalizing, has been proposed to be a core cognitive domain underlying much of human social behavior (Corballis \& Lea, 1999). Moreover, language functions as the primary channel through which social behavior is mediated (Carpenter et al., 1998; Clark, 1996; Tylen et al., 2010). As such, a complete understanding of either relies on an analysis of their interaction.

As a core cognitive domain, it is plausible that mentalizing has been subject to tight constraints imposed by natural selection. In line with this assumption, research on adult mentalizing has found relative homogeneity in neurotypical adult populations (e.g., Castelli et al., 2000; Senju et al., 2009). Such findings have bolstered claims suggesting there are few, if any, meaningful differences in adult mentalizing ability across both individuals and cultures. However, these same findings often come from primarily Western samples (see Liu et al., 2008 and Heyes \& Frith, 2014 for exceptions), employ tests of mentalizing that show ceiling effects in adults (although see Turner \& Felisberti, 2017 for a review of methods appropriate for adults) and equivocate competence and performance (cf. Wu \& Keysar, 2007 for an exception). While human beings may have an implicit and species-typical capacity, or competence, to impute others' mental states, the way in which such imputations structure social behavior may differ across populations in accordance with prevailing socioecological conditions.

An emerging anthropological literature lends credence to this claim. Ethnographic accounts suggest that many small-scale societies have social norms that restrict talking about others' mental states (Robbins \& Rumsey, 2008). Given the documented importance of mental-state talk for children's early sociocognitve development (Ruffman, Slade, \& Crowe, 2002), these accounts, if true, raise a number of questions central to the evolution of language. Does the way in which 
people talk about mental states actually vary across cultures and individuals? How does this variation relate to a presumably universal competence? To what extent does making knowledge of others' mental states explicit facilitate cooperation and communication? Despite the centrality of these questions, few quantitative studies of mental-state talk in a society with such norms have been conducted. Thus, we measured mental state language in a small-scale society with implicit norms against attributing mental states to others. An explicit quantification of mentalstate talk provides a metric against which ethnographic accounts can be measured. Should the data suggest differences in patterns of mental-state talk, subsequent studies are warranted. However, it is critical to first assess the truth of the claims.

A simple response-elicitation task was administered in Spanish to bilingual Shuar / Spanish speakers in a small-scale, hunter-horticulturalist society in Amazonian Ecuador ( $\mathrm{N}=40,20$ female) as well as in English to a sample of American undergraduates ( $\mathrm{N}=20,15$ female). Participants were shown a set of eight silent videos. Each set contained six videos designed to elicit mental state language (MS) and two videos that served as controls (C). MS videos depicted naturalistic interactions between multiple agents and featured richly mentalistic content, such as one character tricking another character. C Videos depicted causal, but inanimate agents interacting. Each video was 30-45 seconds long.

After each video, participants described what happened in the scene. Participants received a minimal prompt to reduce task demands and capture variation in the extent to which mental states were reported. Shuar participants were expected to describe scenes using less mentalizing language than American participants. Verbal responses were transcribed and coded according to a scheme adapted from Castelli et al. (2000) and Ruffman et al. (2002). Word counts for affective states, desire, epistemic states, and perception were obtained. Counts per description were scaled by description length to control for differences in verbosity. Hierarchical Poisson Regression models of word counts were run with culture and video type as fixed factors and participant as a random factor.

American participants were found to use words attributing perception, affective states, and epistemic states to characters significantly more often than were Shuar participants, while Shuar participants used desire words more frequently. These data confirm that our Shuar participants were less likely than Western participants to describe scenes they had witnessed in terms of an agent's mental states. These data are important for a complete understanding of the evolution of language. Language cannot be understood as apart from the mentalizing capacity from which it follows - the two are inextricably linked and undergird human beings' uniquely elaborated sociality. 


\section{References}

Carpenter, M., Nagell, K., Tomasello, M., Butterworth, G., \& Moore, C. (1998). Social cognition, joint attention, and communicative competence from 9 to 15 months of age. Monographs of the society for research in child development, i-174.

Castelli, F., Happé, F., Frith, U., \& Frith, C. (2000). Movement and mind: a functional imaging study of perception and interpretation of complex intentional movement patterns. Neuroimage, 12(3), 314-325.

Clark, H. H. (1996). Using language. Cambridge university press.

Corballis, M. C., \& Lea, S. E. (1999). The descent of mind: Psychological perspectives on hominid evolution. Oxford University Press

Heyes, C. M., \& Frith, C. D. (2014). The cultural evolution of mind reading. Science, 344(6190), 1243091.

Robbins, J., \& Rumsey, A. (2008). Introduction: Cultural and linguistic anthropology and the opacity of other minds. Anthropological Quarterly, 81(2), 407-420

Ruffman, T., Slade, L., \& Crowe, E. (2002). The relation between children's and mothers' mental state language and theory-of-mind understanding. Child development, 73(3), 734-751.

Senju, A., Southgate, V., White, S., \& Frith, U. (2009). Mindblind eyes: an absence of spontaneous theory of mind in Asperger syndrome. Science, 325(5942), 883-885.

Turner, R., \& Felisberti, F. M. (2017). Measuring Mindreading: A review of behavioral approaches to testing cognitive and affective mental state attribution in neurologically typical adults. Frontiers in psychology, 8 .

Tylén, K., Weed, E., Wallentin, M., Roepstorff, A., \& Frith, C. D. (2010). Language as a tool for interacting minds. Mind \& Language, 25(1), 3-29.

Wu, S., \& Keysar, B. (2007). The effect of culture on perspective taking. Psychological science, 18(7), 600-606. 